Research Paper

\title{
Ulinastatin Preconditioning Attenuates Inflammatory Reaction of Hepatic Ischemia Reperfusion Injury in Rats via High Mobility Group Box I(HMGBI) Inhibition
}

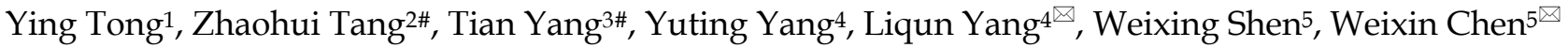 \\ 1. Department of Liver Surgery, Renji Hospital, Shanghai Jiaotong University, School of Medicine, 1630 Dongfang Road, Shanghai 200127, China \\ 2. Department of General Surgery, Xinhua Hospital, Shanghai Jiaotong University, School of Medicine, 1665 Kongiiang Road, Shanghai, 200092, \\ China \\ 3. Department of Hepatic Surgery, Eastern Hepatobiliary Surgery Hospital, the Second Military Medical University, 225 Changhai Road, Shanghai \\ 200438, China \\ 4. Department of Anesthesia and Intensive Care, Eastern Hepatobiliary Surgery Hospital, the Second Military Medical University, 225 Changhai \\ Road, Shanghai 200438, China \\ 5. Department of General Surgery, Qingpu Branch of Zhongshan Hospital, Fudan University, Shanghai, 201700, China
}

\# Drs Tong Y, Tang Z and Yang T contributed equally to this work.

$\triangle$ Corresponding author: Dr. Weixin Chen, Department of General Surgery, Qingpu Branch of Zhongshan Hospital, Fudan University, Shanghai, 201700, China. E-mail: lqyang72721@icloud.com or Dr. Liqun Yang, M.D., Department of Anesthesia and Intensive Care, Eastern Hepatobiliary Surgery Hospital, the Second Military Medical University, 225 Changhai Road, Shanghai 200438, China. E-mail: lqyang72721@hotmail.com.

( ) Ivyspring International Publisher. This is an open-access article distributed under the terms of the Creative Commons License (http://creativecommons.org/ licenses/by-nc-nd/3.0/). Reproduction is permitted for personal, noncommercial use, provided that the article is in whole, unmodified, and properly cited.

Received: 2013.10.10; Accepted: 20I4.0I.15; Published: 20I4.02.I I

\begin{abstract}
Objective It has been found that ulinastatin (UTI) can attenuate hepatic injury in a rat model of ischemia reperfusion (IR), but the specific mechanism is unclear. This study aims to investigate possible pathomechanism of ulinastatin in reducing the inflammatory response after hepatic IR.

Methods A male sprague-dawley(SD) rat model of hepatic ischemia reperfusion injury was used. The rats were randomly divided into 4 groups on average, which were $0.9 \%$ saline and IR group as control, ulinastatin preconditioning (UPC) group, UPC+rHMGBI (recombinant HMGBI) group and UPC +anti-HMGBI group. Serum aminotransferases, TNF- $\alpha$, IL-I and Myeloperoxidase (MPO) levels were measured. Histopathology examination and apoptotic cell detection and the different expression of HMGBI protein were also assessed.

Results Serum levels of aminotransferases, cytokines and hepatic MPO in UPC and UPC+anti-HMGBI groups were significantly lower than those in control group $(p<0.05)$.=Decreased histologic damage and apoptosis were also seen in these two groups $(p<0.05)$.

Conclusions HMGBI expressions in UPC and UPC+anti-HMGBI groups were significantly lower than those in the two control groups $(p<0.05)$, pretreatment with ulinastatin attenuated liver IR injury by reducing HMGBI expression through its anti-inflammatory effects.
\end{abstract}

Key words: Ulinastatin, Preconditioning, High mobility group box 1 protein (HMGB1), Ischemia reperfusion

\section{Introduction}

Ulinastatin (UTI) can attenuate hepatic injury in a rat ischemia reperfusion model and it can reduce reactive oxygen species (ROS) production as reported before. [1] Ulinastatin plays a role in further reduced liver reperfusion injury after liver cell necrosis and apoptosis through the above-mentioned an- 
ti-inflammatory and antioxidant effects [2], but the specific pathway and mechanism remain unclear.

HMGB1 is an important endogenous injury-related molecule, widely distributed in mammalian lymphoid tissues, brain, liver, lung, heart, spleen, kidney and other tissues, which exists in the nucleus in most organizations, whereas it mainly exists in cytoplasm in liver and brain tissues [3]. HMGB1 expression in the physiological state is maintained at an extremely low level [4]. Previous study confirmed that HMGB1 mediated endotoxin induced sepsis, and was also a late lethal inflammatory mediators [5]. Whereas the biological effects of HMGB1 in different organs in the body are similar, and ulinastatin can inhibit the release of TNF- $a$, IL-1, cytokines and other inflammatory mediators, [6] we speculate that ulinastatin may inhibit cytokine-induced HMGB1 expression, thereby reducing the inflammatory response after hepatic ischemia-reperfusion. It has not been reported that ulinastatin can inhibit ROS releasing from Kupffer cells and other non-primary interstitial cells, which induces free radical damage in early reperfusion stage, thus further reducing the expression of HMGB1 release

\section{Materials and Methods}

\section{Experimental animals}

Male Sprague-Dawley rats (12-14 weeks, 250-300 g) from the Experimental Animal Center, the Chinese National Institute of Medicine (Shanghai, China) were used. Animals were housed at the air conditioning kept indoors, room temperature $22-25^{\circ} \mathrm{C}$. Animals were allowed free access to food and water until 12 hours prior to the experiments as fasting.

\section{Animal model}

The Animal Care and Scientific Committee of the Second Military Medical University (SMMU) approved the experimental protocol. A model of segmental (70\%) hepatic ischemia was used as previously described [7]. After a midline laparotomy under anesthesia with sodium pentobarbital $(40 \mathrm{mg} / \mathrm{kg}$, i.p.), the ligaments around the liver were disconnected. The hepatic artery, portal vein, and bile duct to the left and median hepatic lobes were carefully revealed, and occluded with an atraumatic microvascular clamp. The clamp was removed 45 mins later to allow reperfusion. This method produces ischemia to the left and median lobes of the liver while leaving the blood supply to the right and caudate lobes uninterrupted. At the end of the ischemia period, the vascular clamp was removed and the liver was reperfused for 2 hours. After reperfusion, the vena cava was opened and 3-5 ml blood was collected in sterile syringes without anticoagulant and centrifuged to separate the serum. The serum samples were stored at $-20^{\circ} \mathrm{C}$ for later batch analysis of hepatic function and cytokine assay. The liver was then perfused with cold saline through the portal vein. Ischemic left hepatic tissue samples were collected and the specimens were: 1 ) fixed in $10 \%$ formalin and embedded in paraffin for histological studies; 2) immediately frozen in isopentane and liquid nitrogen, then stored at $-80^{\circ} \mathrm{C}$ for later analysis. The animals were then given a further dose of pentobarbitone $(50 \mathrm{mg}$ ) before being killed by exsanguination.

\section{Experimental groups}

In addition to a sham operation group, SD rats were randomly divided into 4 groups $(n=10$ in each group). The first group received a standard ischemia-reperfusion regimen, and was served as a positive control. IR model was established, whereas $0.9 \%$ sodium chloride was infused at $6 \mathrm{ml} / \mathrm{kg}$ through the tail vein $5 \mathrm{~min}$ before ischemia. All the doses selected below were described in previous study $[2,8,9]$ and our preliminary animal experiments. Animals in ulinastatin pretreatment group (UPC) were injected 20 $\mathrm{IU} / \mathrm{ml}$ of $1.5 \mathrm{ml} / \mathrm{kg}$ of ulinastatin (Techpool Bio Pharmaceutical Co., Ltd. Guangzhou, China) $5 \mathrm{~min}$ before ischemia through the tail vein, and then proceed to ischemia and reperfusion. In HMGB1 induced group (UPC + rHMGB1), animals were administered intraperitoneally $10 \mu \mathrm{g} \cdot \mathrm{kg}^{-1}$ of rHMGB1 antibody (Sino Biological Inc. Beijing, China) one hour before occlusion, and then underwent the same procedure as those in UPC group. 100 $\mu$ g of primary rabbit-anti-rat HMGB1 polyclonal antibody (Anti-HMGB1, Abcam, NV, USA) was injected intraperitoneally one hour before clamping in UPC+Anti-HMGB1group, and the other steps were same as the UPC group.

\section{Measurement of Serum transaminases, cytokines, and hepatic myeloperoxidase}

Alanine aminotransferase (ALT) and aspartate aminotransferase (AST) levels in serum were determined with an autoanalyzer (Model 7600, Hitachi Co.; Tokyo, Japan). 4-5ml of fresh blood was drawn off, and kept at $37^{\circ} \mathrm{C}$ for $30 \mathrm{mins}, 4^{\circ} \mathrm{C}$ for two hours and after centrifuged for $15 \mathrm{mins}$ at $3000 \mathrm{rpm}$, the upper serum 200 $\mathrm{\mu l}$ was drawn off and installed in EP tube carefully. TNF-a and IL-1were measured by enzyme-linked immunosorbent assay (ELISA) kit box (Biosource International, camarillo, CA, USA). Liver homogenates were prepared by immediate centrifugation at $8,000 \mathrm{~g}$ for 10 minutes, myeloperoxidase (MOD) activity as lipid peroxidation determination in the supernatant was assessed by biochemical kits purchased commercially (Nan-Jing Jiancheng Biochemicals LTD, China). 


\section{Morphological observation and apoptotic cell detection}

Liver samples were excised from the anterior edge of the left lobe 120 mins after reperfusion. Small portions $(0.5 \mathrm{~cm} \times 0.5 \mathrm{~cm})$ were fixed immediately in $10 \%$ buffered paraformaldehyde ( $\mathrm{pH}$ 7.2) and embedded in paraffin. These portions were cut into $4-\mu \mathrm{m}$ thick sections and stained with hematoxylin and eosin (H\&E). High-powered microscopy $(1 \times 200)$ was used to examine theses sections for the following signs of liver injury: condensation of nuclei (nuclear pyknosis), loss of hepatocellular borders, areas of necrosis, The score was determined by dividing the measured necrotic area by the total area of the field using Image-Pro-Plus $^{\circledR}$ Software (Media Cybernetics Inc, Bethesda, MD). On the other hand, all groups of paraffin sections for liver biopsies were detected for apoptosis cells by the transferase-mediated deoxyutidine triphosphate (dUTP) nick-end labeling method (TUNEL) (In Situ Cell Detection Kit, Roche Biochemicals, Mannheim, Germany). To quantify apoptosis, the number of TUNEL positive cells was counted in 8 random microscopic fields and quantified.

\section{Immunohistochemistry of HMGB I protein in liver tissues}

The fixed liver block was embedded in paraffin and sectioned into $5 \mu \mathrm{m}$ slices. After de-paraffinage and re-hydration, the sections $(5 \mu \mathrm{m})$ were dipped into an antigen retrieval buffer ( $10 \mathrm{mM}$ sodium citrate; $\mathrm{pH}$ 6.0) and underwent warm retrieval in a microwave oven at $95-100^{\circ} \mathrm{C}$ for 5 mins and then incubated at room temperature with $3 \%$ hydrogen peroxide to deactivate endogenous peroxidases. Nonspecific reactivity was blocked using $2 \%$ bovine serum albumin (BSA) at room temperature for 30 mins. This was followed by incubating the primary antibody rabbit-anti-rat to HMGB1 antibody (dilution 1:100; Abcam Inc, San Francisco, CA), overnight at $4^{\circ} \mathrm{C}$. After washing with phosphate-buffered saline (PBS), a polymer enhancer and a polymerized anti-rabbit or anti-mouse immunoglobulin G (IgG) (dilution 1:200, Jingmei, Shanghai, China) labelled with horseradish peroxidase was applied. Antibodies were visualized as buffy granules in the cytoplasma using a DAB kit (Maixin Biological Technology, Fujian, China). Area density of HMGB1 positive tissues were analysed in 6 random high powered $(1 \times 400)$ microscopic fields using Image-Pro-Plus ${ }^{\circledR}$ Software.

\section{Statistical analyses}

Data analysis was performed using the Prism 4.0 statistical software package (Graph-Pad Software, San Diego, CA). Data were expressed as mean \pm SEM. Analysis of variance (ANOVA) with Bonferroni's multiple comparisons test was used to compare values among all groups. Statistical differences were considered significant if the $p$ value was less than 0.05 .

\section{Results}

\section{Serum aminotransferases, cytokines, and hepatic myeloperoxidase}

As shown in Figure 1, serum ALT and AST levels significantly decreased in UPC group and UPC+Anti-HMGB1 group compared with control group $(p<0.05)$. However, there was no significant difference between control and UPC+rHMGB1 group $(p>0.05)$. Serum ALT and AST levels in UPC group were comparable to those in UPC+Anti-HMGB1 group $(\mathrm{p}>0.05)$. Significantly increasing serum cytokines TNF- $\alpha$ and IL-1 levels were found in the control group and the UPC+rHMGB1 group compared with UPC group and UPC+Anti-HMGB1 group $(\mathrm{p}<0.05)$, but the differences between control group and the UPC + rHMGB1 group were not statistically significant $(p>0.05)$. The UPC+Anti-HMGB1 group had similar serum cytokines TNF- $\alpha$ and IL-1 levels compared with UPC group ( $p>0.05)$. At 120 mins after reperfusion, rats pretreated with UPC and UPC plus Anti-HMGB1 had significantly reduced levels of MDA in liver homogenates compared to those in control.

\section{Morphological observation and apoptotic cell detection}

As shown in Figure 2, At 120 mins after reperfusion, the liver histology in ulinastatin pretreated group and UPC plus Anti-HMGB1 group exhibited fewer and smaller areas of necrosis, sinusoidal dilation and structural derangement around the central vein as compared to the control and UPC+rHMGB1 groups. The amount of apoptosis and the number of TUNEL-positive cells among UPC and UPC+ Anti-HMGB1 groups were less than that in the control and UPC+rHMGB1 groups.

\section{Immunohistochemistry of HMGB I expression in liver}

As shown in Figure 3, the expression of HMGB1 in liver cells significantly decreased in UPC and UPC+Anti-HMGB1 groups compared with the control and UPC + rHMGB1 group $(\mathrm{p}<0.05)$. 
A

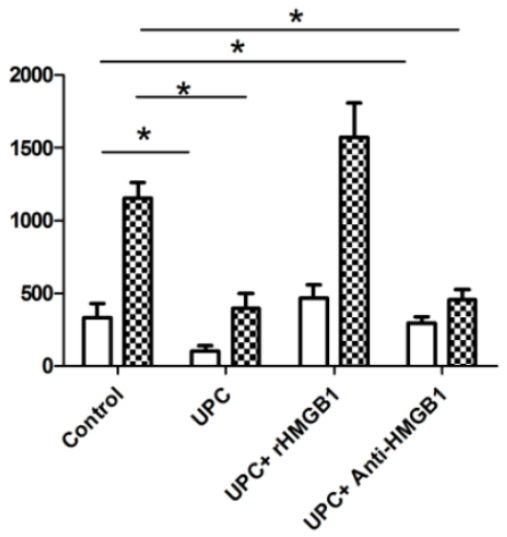

C

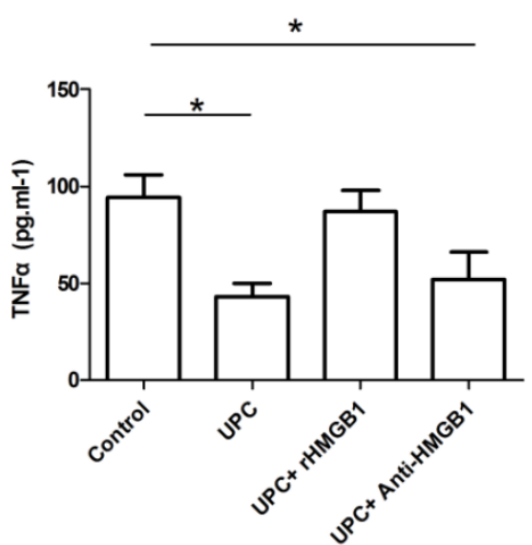

ALT (IU.I-1)

$\mathbf{\infty}$ AST (IU.L-1)

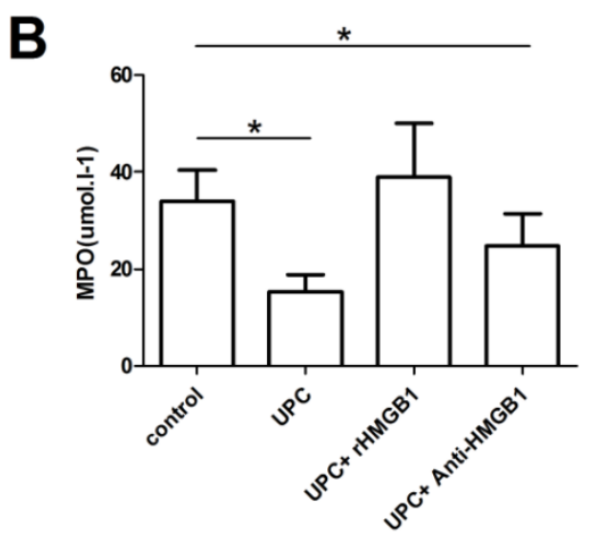

D

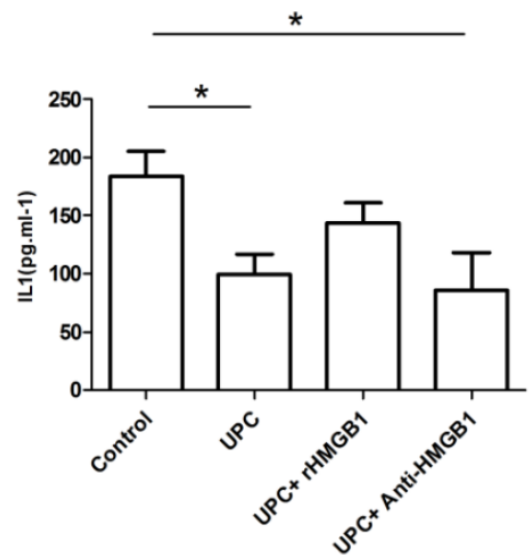

Figure I. Serum aminotransferases, cytokines, and hepatic myeloperoxidase among different groups. Figure A showed serum ALT and AST levels significantly increased when $20 \mathrm{IU} / \mathrm{ml}$ of $\mathrm{l} .5 \mathrm{ml} / \mathrm{kg}$ of ulinastatin pretreated (UPC) group or combined with I00 $\mu \mathrm{g}$ of anti-HMGBI (UPC+Anti-HMGB I group) compared to those in control $(p<0.05)$, but there was no significant difference between control group and UPC+rHMGBI group ( $>>0.05)$. As showed in figure $B$ and $C$, significantly increasing serum cytokines TNF- $\alpha$ and IL-I levels were found in the control group and the UPC+rHMGBI group compared with UPC group and UPC+Anti-HMGBI group $(\mathrm{p}<0.05)$. At 120 min after reperfusion, rats in UPC and UPC plus Anti-HMGBI had significantly reduced levels of myeloperoxidase (MDA) in liver homogenates compared to those in control as showed in figure $D$. Values are mean \pm SD of 10 animals in each group. $* \mathrm{P}<0.05$ vs. Control.
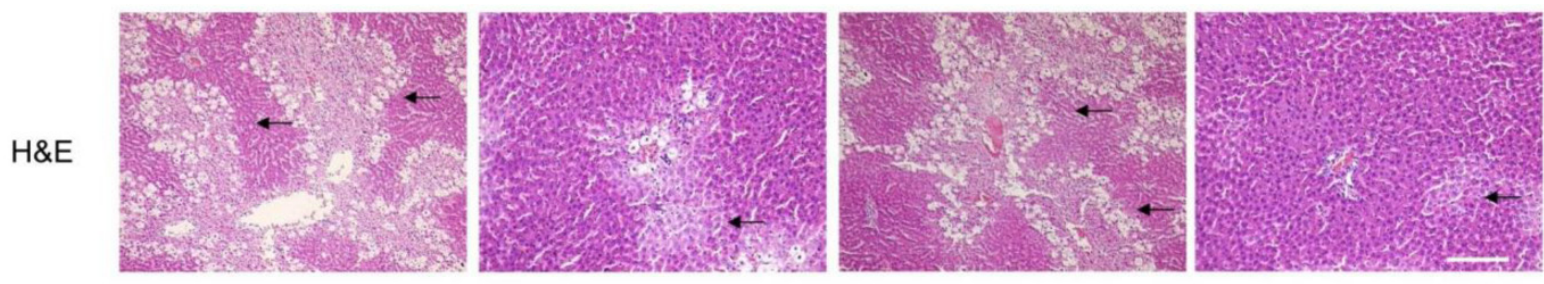

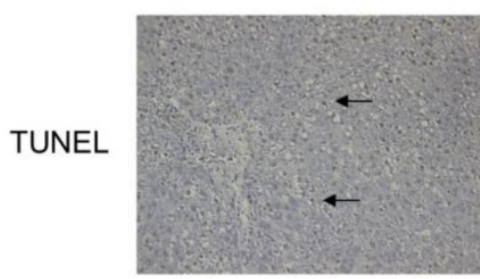

Control

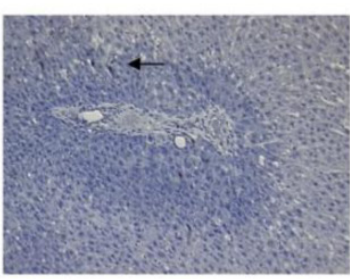

UPC

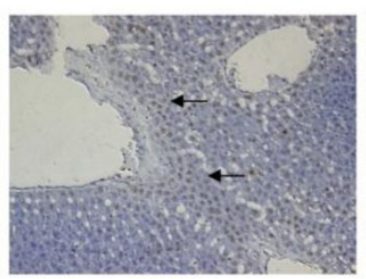

UPC+rHMGB1

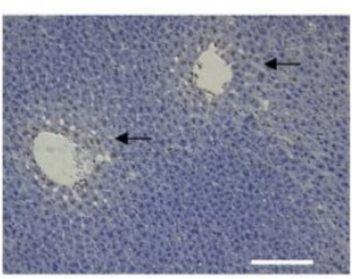

UPC+Anti-HMGB1

Figure 2. Effect of ulinastatin preconditioning (UPC) on attenuating liver ischemia reperfusion injury. Liver tissue was processed with $\mathrm{H} \& \mathrm{E}$ staining for light microscopy. Photograph depicts typical pattern of focal necrosis (black arrows) after ischemic degeneration of hepatocytes around the central venous area. Areas of necrosis were significantly lower in UPC and Anti-HMGBI treated groups than in control. Magnification: 200×. Scale bar = $200 \mu \mathrm{m}$. Hepatocyte apoptosis was determined by immunohistochemical analysis by microscopy after TUNEL staining. Photograph depicts typical pattern of apoptotic cells (black arrows). Percentage of apoptotic cells was significantly lower in UPC and Anti-HMGBI treated groups than in control group. Magnification: $200 \times$. Scale bar $=200 \mu \mathrm{m}$. 

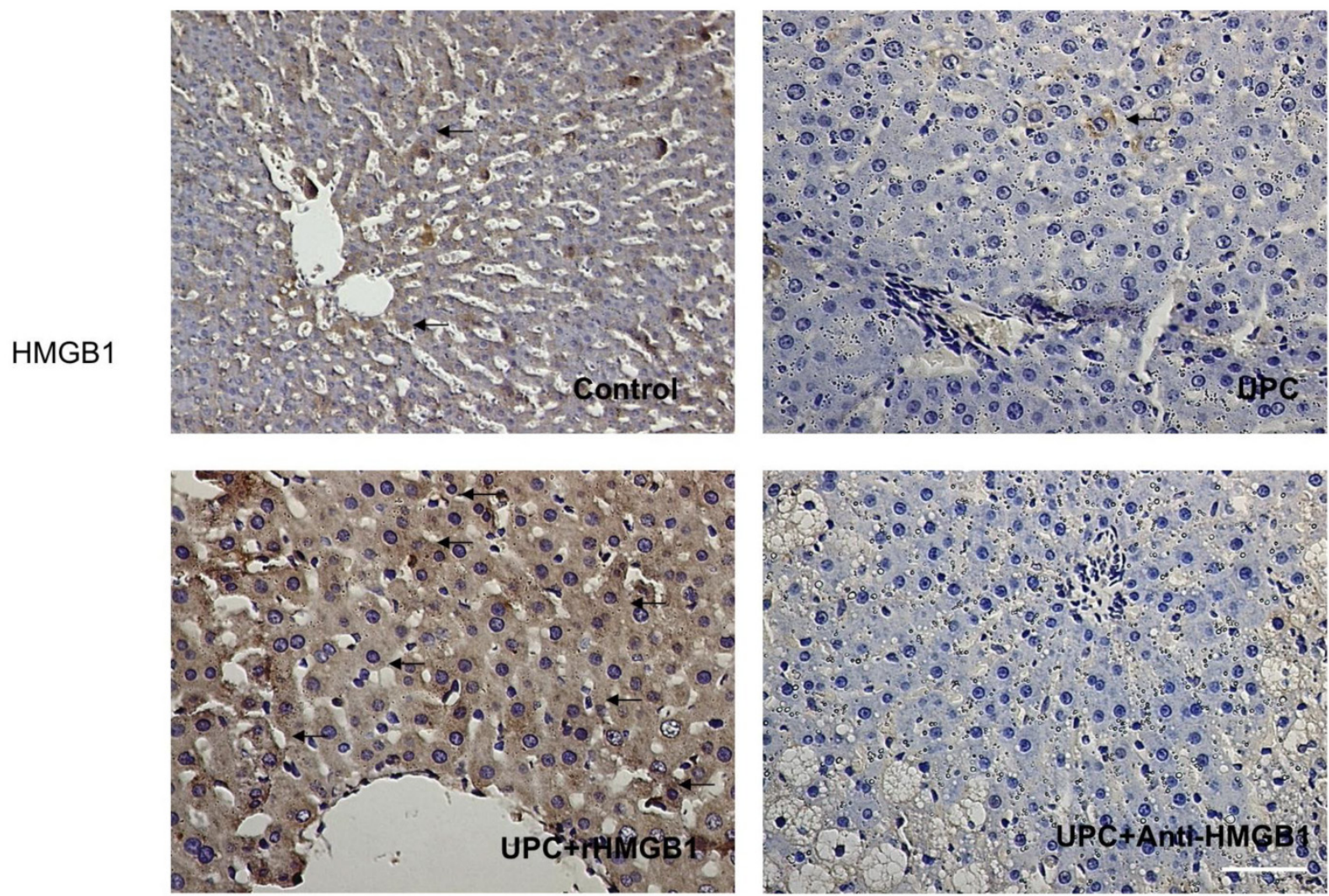

Figure 3. Immunohistological result of HMGB I protein expression in groups. Hepatic HMGBI positive cells were defined as stained with brown in cytoplasm (black arrows). It showed a decrease in HMGBI expression in UPC and Anti-HMGBI groups compared with control. Magnification: 400x. Scale bar $=100 \mu \mathrm{m}$

\section{Discussion}

Results from our study demonstrated that pretreatment with ulinastatin was associated with inhibiting cytokine-induced HMGB1 expression, thereby reducing the inflammatory response after hepatic IR, while maintaining the structure of the hepatic parenchyma and suppressing hepatocytes apoptosis and serum levels of aminotransferases, suggesting that UPC had hepatoprotective effects. Hepatic ischemia and reperfusion injury can happen in many situations, including reduced cardiac function and liver surgery. Maintaining liver function remains to be an important clinical task [10-12], while reduced liver function after hepatic IR reflected poor prognosis. The present results shown herein suggested that ulinastatin preconditioning may protect the ischemic liver during various clinical conditions.

A role for the nuclear protein, HMGB1, in the pathogenesis of I/R-induced injury in a number of tissues, including the liver, has been proposed [3]. And recent evidence now exists to shed light on the vicious ROS-HMGB1 loop in IR injury [9]. Extracellular HMGB1 induces receptor for advanced glycation end products (RAGE) signalling which induces elevated free radicals [13], especially superoxide anion radical $\left(\mathrm{O}_{2}-\right)$ from which the generation of many ROS derives [14], followed by HMGB1 activation of NADPH oxidase and increased ROS production in a toll-like receptor 4 (TLR4)-dependent manner [5]. The TLR4-dependent ROS production can promote the hypoxia-induced HMGB1 released by hepatocytes [15]. Mutual promotion between ROS and HMGB1 forms a vicious circuit. UTI was reported to inhibit the extracellular release of toxic free radicals by neutrophils via the suppression of NADPH oxidase $[16,17]$ and might interrupt the vicious ROS-HMGB1 loop [9]. In addition to the formation of free radicals, IR damage in the liver consists of two additional factors, namely inflammatory response and microcirculation. The inflammatory response plays an important role in homeostasis after IR. Because of the strategic localization of the liver in blood supply to the entire gastrointestinal tract, ischemia of the liver invariably brings about an influx of bacteria and associated toxins on reperfusion [18, 19]. Sample evidences have demonstrated that the massive inflammatory response is also a major cause of hepatic injuries after the IR [20,21]. In our experiments, TNF- $\alpha$ and IL-1 of UPC rats decreased significantly after the IR, which suggested that hepatoprotective effects of UPC indeed partly mediated by the inhibition of inflammatory respons- 
es. Considering HMGB1 acting as a pro-inflammatory cytokine in stimulating the release of other cytokines, the inhibited effects of inflammatory responses of UPC can be partly explained by the indirect suppression of TNF-a and IL-1 via HMGB1 inhibition.

It has been reported that expression of HMGB1 promotes micro-vascular thrombosis in a model of thrombin-induced disseminated intravascular coagulation in rats $[22,23]$ and HMGB1 may have contributed to the reduced liver reperfusion by promoting fibrin deposition in the hepatic micro-vessels [24]. We did not directly examine microcirculation in this study. Microscopic examination of hepatic slices, however, indicated that the improved microcirculation might be one of the mechanisms of UPC's hepatoprotective effects through which HMGB1 suppression alleviates the IR damage.

There are various types of cell death, including necrosis, autophagy, and apoptosis. It has been reported that TNF-a can directly impair mitochondrial activity, causing apoptosis and necrosis [25], and HMGB1 was important for oxidative stress-mediated autophagy [26]. The expressions of HMGB1 and TNF- $\alpha$ are regulated by NF-kB [27], and UPC could suppress NF- $\mathrm{KB}$ activation. Our results suggested that UPC inhibited hepatic damage after hepatic IR injury [1]. We speculate that the inhibition of hepatic cell death might be related to HMGB1 and TNF-a inhibited by UPC through down-regulating NF-kappa B activation.

Two unexpected results appeared in the present study. Firstly, no superimposed effects were found in the UPC+Anti-HMGB1 group compared with UPC group, as both UPC and Anti-HMGB1 had hepatoprotective effects. This result can be elucidated by UPC and Anti-HMGB1 having the same target in protecting against hepatic IR injury. It demonstrated that UTI preconditioning attenuates hepatic IR injury in Rats by inhibiting the expression of HMGB1. Secondly, antagonistic effects were found in UPC + rHMGB1 group compared with UPC group, although the protective effect of HMGB1 preconditioning in hepatic IR injury has been reported [28]. The HMGB1 tolerant state following intravenous rHMGB preconditioning may be mediated by two distinct mechanisms: an early transient cellular refractory state and a later production of antibody which assists the reticuloendothelial system with clearance and destruction of the HMGB1 [29]. The surmise that ulinastatin and rHMGB1can coagulate into an inactive conjugate to eliminate them in serum may be an acceptable explication for the antagonistic effects of UTI and rHMGB1. However, further investigations in vivo are needed to clarify this important issue.

Several limitations to this study are worth not- ing. Firstly, this study used a rat model of hepatic IR injury; therefore the results may not reflect what occurs in humans. Secondly, the experiments focus on evaluating the short-term effect of ulinastatin preconditioning, and further studies are needed to determine its long-term efficacy. Finally, the effects of ulinastatin post-IR injury were not evaluated in our study. Additional research is needed to address these issues.

In summary, our results confirmed that ulinastatin preconditioning could effectively protect the liver from IR injuries, and decrease the expression of HMGB1 in hepatocytes. It also demonstrated that the protective effects of UPC, as a "magic bullet" targeting inflammatory sites, against the liver IR damage are mediated by the prohibition of HMGB1. Based on present findings, although HMGB1 is almost undoubtedly one piece of the puzzle as opposed to a panacea, HMGB1 is believed to be a promising molecular target in developing novel treatment and prevention for hepatic IR injuries.

\section{Acknowledgements}

We gratefully acknowledge the National Natural Science Foundation Committee of China (Grant No. 81272066), Rising-Star Fellow-up program of Shanghai Science and Technology Commission (Grant No.12QH1402800) and Techpool Research Foundation for their financial support.

\section{Competing Interests}

The authors have declared that no competing interest exists.

\section{References}

1. Aihara T, Shiraishi M, Hiroyasu S, Hatsuse K, Mochizuki H, Seki S, et al. Ulinastatin, a protease inhibitor, attenuates hepatic ischemia/reperfusion injury by downregulating TNF-alpha in the liver. Transplant Proc. 1998; 30: 3732-4. doi:S0041-1345(98)01214-7 [pii].

2. Yang YL, Li JP, Xu XP, Dou KF, Yue SQ, Li KZ. Protective effects of tumor necrosis factor alpha antibody and ulinastatin on liver ischemic reperfusion in rats. World J Gastroenterol. 2004; 10: 3161-4.

3. Klune JR, Dhupar R, Cardinal J, Billiar TR, Tsung A. HMGB1: endogenous danger signaling. Mol Med. 2008; 14: 476-84. doi:10.2119/2008-00034.Klune.

4. Kang R, Livesey KM, Zeh HJ, 3rd, Lotze MT, Tang D. HMGB1 as an autophagy sensor in oxidative stress. Autophagy. 2011; 7: 904-6. doi:15704 [pii].

5. Fan J, Li Y, Levy RM, Fan JJ, Hackam DJ, Vodovotz Y, et al. Hemorrhagic shock induces $\mathrm{NAD}(\mathrm{P}) \mathrm{H}$ oxidase activation in neutrophils: role of HMGB1-TLR4 signaling. J Immunol. 2007; 178: 6573-80.

6. Tang D, Shi Y, Kang R, Li T, Xiao W, Wang H, et al. Hydrogen peroxide stimulates macrophages and monocytes to actively release HMGB1. J Leukoc Biol. 2007; 81: 741-7. doi:10.1189/jlb.0806540.

7. Serafin A, Rosello-Catafau J, Prats N, Gelpi E, Rodes J, Peralta C. Ischemic preconditioning affects interleukin release in fatty livers of rats undergoing ischemia/reperfusion. Hepatology. 2004; 39: 688-98. doi:10.1002/hep.20089.

8. Tsung A, Sahai R, Tanaka H, Nakao A, Fink M, Lotze M, et al. The nuclear factor HMGB1 mediates hepatic injury after murine liver ischemia-reperfusion. J Exp Med. 2005; 201: 1135-43. doi:10.1084/jem.20042614.

9. Tanaka R, Fujita M, Tsuruta R, Fujimoto K, Aki HS, Kumagai K, et al. Urinary trypsin inhibitor suppresses excessive generation of superoxide anion radical, systemic inflammation, oxidative stress, and endothelial injury in endotoxemic rats. Inflamm Res. 2010; 59: 597-606. doi:10.1007/s00011-010-0166-8.

10. Wobbes T, Bemelmans BL, Kuypers JH, Beerthuizen GI, Theeuwes AG. Risk of postoperative septic complications after abdominal surgical treatment in 
relation to perioperative blood transfusion. Surg Gynecol Obstet. 1990; 171: 59-62.

11. Cowan RE, Jackson BT, Grainger SL, Thompson RP. Effects of anesthetic agents and abdominal surgery on liver blood flow. Hepatology. 1991; 14: 1161-6.

12. Bahde R, Spiegel H. Hepatic ischaemia-reperfusion injury from bench to bedside. Br J Surg. 2010; 97: 1461-75. doi:10.1002/bjs.7176.

13. Ding Y, Kantarci A, Hasturk H, Trackman PC, Malabanan A, Van Dyke TE. Activation of RAGE induces elevated O2- generation by mononuclear phagocytes in diabetes. J Leukoc Biol. 2007; 81: 520-7. doi:10.1189/jlb.0406262.

14. Victor VM, Rocha $M$, De la Fuente M. Immune cells: free radicals and antioxidants in sepsis. Int Immunopharmacol. 2004; 4: 327-47. doi:10.1016/j.intimp.2004.01.020.

15. Tsung A, Klune I, Zhang X, Jeyabalan G, Cao Z, Peng X, et al. HMGB1 release induced by liver ischemia involves Toll-like receptor 4 dependent reactive oxygen species production and calcium-mediated signaling. J Exp Med. 2007; 204: 2913-23. doi:10.1084/jem.20070247.

16. Nishijima J, Hiraoka N, Murata A, Oka Y, Kitagawa K, Tanaka N, et al. Protease inhibitors (gebexate mesylate and ulinastatin) stimulate intracellular chemiluminescence in human neutrophils. J Leukoc Biol. 1992; 52: 262-8.

17. Kudo Y, Egashira T, Yamanaka Y. Protective effect of ulinastatin against liver injury caused by ischemia-reperfusion in rats. Jpn J Pharmacol. 1992; 60: 239-45.

18. Wunder C, Potter RF. The heme oxygenase system: its role in liver inflammation. Curr Drug Targets Cardiovasc Haematol Disord. 2003; 3: 199-208.

19. Alcaraz MJ, Fernandez P, Guillen MI. Anti-inflammatory actions of the heme oxygenase-1 pathway. Curr Pharm Des. 2003; 9: 2541-51.

20. Soares MP, Brouard S, Smith RN, Bach FH. Heme oxygenase-1, a protective gene that prevents the rejection of transplanted organs. Immunol Rev. 2001; 184: 275-85.

21. Wagener FA, Volk HD, Willis D, Abraham NG, Soares MP, Adema GJ, et al. Different faces of the heme-heme oxygenase system in inflammation. Pharmacol Rev. 2003; 55: 551-71. doi:10.1124/pr.55.3.5.

22. Hatada T, Wada H, Nobori T, Okabayashi K, Maruyama K, Abe Y, et al. Plasma concentrations and importance of High Mobility Group Box protein in the prognosis of organ failure in patients with disseminated intravascular coagulation. Thromb Haemost. 2005; 94: 975-9. doi:10.1160/TH05-05-0316.

23. Ito T, Kawahara K, Nakamura T, Yamada S, Abeyama K, Hashiguchi T, et al. High-mobility group box 1 protein promotes development of microvascular thrombosis in rats. J Thromb Haemost. 2007; 5: 109-16. doi:10.1111/j.1538-7836.2006.02255.x.

24. Mabuchi A, Wake K, Marlini M, Watanabe H, Wheatley AM. Protection by glycyrrhizin against warm ischemia-reperfusion-induced cellular injury and derangement of the microcirculatory blood flow in the rat liver. Microcirculation. 2009; 16: 364-76. doi:10.1080/10739680902796917.

25. Hatano E. Tumor necrosis factor signaling in hepatocyte apoptosis. J Gastroenterol Hepatol. 2007; 22 Suppl 1: S43-4. doi:10.1111/j.1440-1746.2006.04645.x.

26. Tang D, Kang R, Livesey KM, Zeh HJ, Lotze MT. High Mobility Group Box 1 (HMGB1) Activates an Autophagic Response to Oxidative Stress. Antioxid Redox Signal. 2011. doi:10.1089/ars.2010.3666

27. Pahl HL. Activators and target genes of Rel/NF-kappaB transcription factors. Oncogene. 1999; 18: 6853-66. doi:10.1038/sj.onc.1203239.

28. Izuishi K, Tsung A, Jeyabalan G, Critchlow ND, Li J, Tracey KJ, et al. Cutting edge: high-mobility group box 1 preconditioning protects against liver ischemia-reperfusion injury. J Immunol. 2006; 176: 7154-8.

29. Greisman SE, Young EJ, Carozza FA, Jr. Mechanisms of endotoxin tolerance. V. Specificity of the early and late phases of pyrogenic tolerance. J Immunol. 1969; 103: 1223-36. 